\title{
A FLEXIBLE BIPOLAR QUERYING APPROACH WITH IMPRECISE DATA AND GUARANTEED RESULTS
}

\author{
SÉBASTIEN DESTERCKE, PATRICE BUCHE, AND VALÉRIE GUILLARD
}

\begin{abstract}
In this paper, we propose an approach to query a database when the user preferences are bipolar (i.e., express both constraints and wishes about the desired result) and the data stored in the database are imprecise. Results are then completely ordered with respect to these bipolar preferences, giving priority to constraints over wishes. Additionally, we propose a treatment that allows us to guarantee that any query will return a result, even if no element satisfies all constraints specified by the user. Such a treatment may be useful when user's constraints are unrealistic (i.e., cannot be all satisfied simultaneously) and when the user desires a guaranteed result. The approach is illustrated on a real-world problem concerning the selection of optimal packaging for fresh fruits and vegetables.
\end{abstract}

\section{INTRODUCTION}

In some applications, there may be a need to differentiate, within queries, between negative preferences and positive ones. Negative preferences correspond to constraints, since they specify which values or object have to be rejected (i.e., those that do not satisfy constraints), while positive preferences correspond to wishes, as they specify which objects are more desirable than others (i.e., satisfy user wishes) without rejecting those that do not meet the wishes.

Indeed, while the first type of preference should be satisfied by query results, satisfying the second type of preference can be considered as optional, as the user does not consider them as necessary requirements. Also, it may be useful to have procedures that send back to the user some answer to its query, even if this answer does not completely fulfil its need (suggestions proposed by modern web search engines when entering mistyped entries can be assimilated to such procedures). Finally, there may be imprecise data in the database, and there is also a need to take this imprecision into account. In this paper, we propose to consider these three problems in a

Key words and phrases. Bipolarity, Possibility theory, Fuzzy databases, Food engineering, Applications. 
0050

0051

0052

0053

0054

0055

0056

0057

0058

0059

0060

0061

0062

0063

0064

0065

0066

0067

0068

0069

0070

0071

0072

0073

0074

0075

0076

0077

0078

0079

0080

0081

0082

0083

0084

0085

0086

0087

0088

0089

0090

0091

0092

0093

0094

0095

0096

0097

0098

common framework, using the notion of bipolar information and of fuzzy pattern matching.

The notions of bipolar preferences and of bipolar information in general have recently received increasing attention [2, 23]. Roughly speaking, information is said to be bipolar when there is a positive and a negative part of the information. These negative and positive parts of the information may have different natures, and should therefore be processed in parallel, and not as a single piece of information. This kind of bipolarity [14], coined as asymmetric, is the one we are concerned with. For example, we may feel both positive and negative about something, without being able to fuse these two feelings in a unique one (for example, eating ice cream gives a gustative pleasure, but one can also feel guilty about it).

In the case of database queries, asymmetric bipolarity is useful to distinguish negative preferences or constraints (i.e. criteria that a satisfying answer must satisfy) from positive preferences or wishes (i.e. criteria that a good answer should satisfy, if possible). For example, in the query "a new car not too expensive and if possible red", "not too expensive" is clearly a requirement while "red" merely expresses a wish.

In this paper, we propose a method to treat bipolar preferences in data bases where data can be uncertain. In particular, this method uses the bipolar nature of preferences to induce an (pre-)ordering between query results, so that priority is given to those instances that are the most likely to satisfy all expressed constraints. Section 2 describes the method, while Section 3 illustrates the approach on a use case coming from a new decision support system (DSS) currently developed in our lab where a (industrial/researcher) user wants to select a packaging material that best suits his/her needs. Finally, we give some elements of comparison with previous works in Section 4 .

\section{METHOD}

This section first recalls some basic tools that will be used in the method, before describing the method itself.

2.1. Preliminaries: fuzzy pattern matching. In this paper, we use fuzzy sets [33] to represent preferences in our queries and possibility distributions [19] to represent the possible imprecision in the data. A normalized fuzzy set $\mu$ over a variable $X$ assuming its value on $\mathscr{X}$ is a mapping $\mu: \mathscr{X} \rightarrow[0,1]$ such that there is at least one $x \in \mathscr{X}$ such that $\mu(x)=1$. Here, we assume that $\mathscr{X}$ is either a finite set of elements (e.g., the colour of a car) or a subset of the real line (e.g., the maximal speed of a car).

Here, fuzzy sets are used to express preferences provided by a user in a query. That is, for a given variable $X$, the fuzzy value $\mu(x)$ expresses 


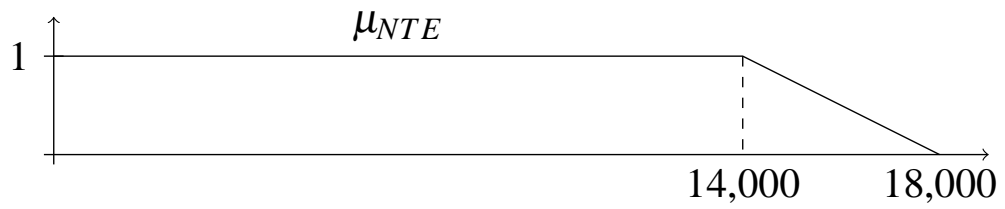

\section{FIGURE 1. Fuzzy set $\mu_{N T E}$ describing "Not Too Expensive"}

to which degree the value $x$ satisfies the preference represented by $\mu$, with $\mu(x)=1$ meaning that the preference is fully satisfied and $\mu(x)=0$ that it is completely unsatisfied.

Example 1. Consider again our car example "a new car not too expensive and if possible red". Assume the user has specified that "Not too expensive" means that any price over $18,000 \$$ is unacceptable, while any price lower than $14,000 \$$ can be considered as totally satisfactory. The corresponding preference is represented by the fuzzy set $\mu_{N T E}$ in Figure 1 . Given this representation, we have, for example, that a price of $15,000 \$$ fulfils the user preferences at a degree $\mu_{N T E}(15,000)=2 / 3$

Possibility distributions, on the other hand, are simple uncertainty representations allowing to model data whose value is imprecisely known. A possibility distribution $\pi$ over a variable $X$ is also a mapping $\pi: \mathscr{X} \rightarrow[0,1]$ such that there is at least one $x \in \mathscr{X}$ such that $\pi(x)=1$. They are therefore equivalent to fuzzy sets from a formal point of view, but possess different semantics. Indeed, they describe our knowledge about the potential value of $X$. Two measures or set-functions can be derived from a possibility distribution, namely the necessity and possibility measures, which are such that, for every event $A \subset \mathscr{X}$,

$$
\Pi(A)=\sup _{x \in A} \pi(x) ; \quad N(A)=\inf _{x \in A^{c}}(1-\pi(x))=1-\Pi\left(A^{c}\right),
$$

where $\Pi(A)$ and $N(A)$ express to which extent it is respectively plausible and certain that the actual value of $X$ lies in A.

Note that possibility distributions can model both precisely known values ( $X=x$ corresponds to the distribution $\pi(x)=1$ and zero everywhere else) and set-valued variables ( $X \in A$ corresponds to the distribution $\pi(x)=1$ if $x \in A$, zero otherwise). In the same ways, fuzzy sets can model crisp preferences (i.e., those used in classical queries).

In the rest of the paper, we consider that each query (or preference) $P$ on an attribute $X$ is expressed by a fuzzy set $\mu_{P}$ (possibly degenerated into a crisp preference) and our knowledge $D$ about the attribute value for a particular tuple is given by a possibility distribution $\pi_{D}$ (also possibly degenerated in a crisp set). Our knowledge about the imprecise evaluation 
0148

0149

0150

0151

0152

0153

0154

0155

0156

0157

0158

0159

0160

0161

0162

0163

0164

0165

0166

0167

0168

0169

0170

0171

0172

0173

0174

0175

0176

0177

0178

0179

0180

0181

0182

0183

0184

0185

0186

0187

0188

0189

0190

0191

0192

0193

0194

0195

0196

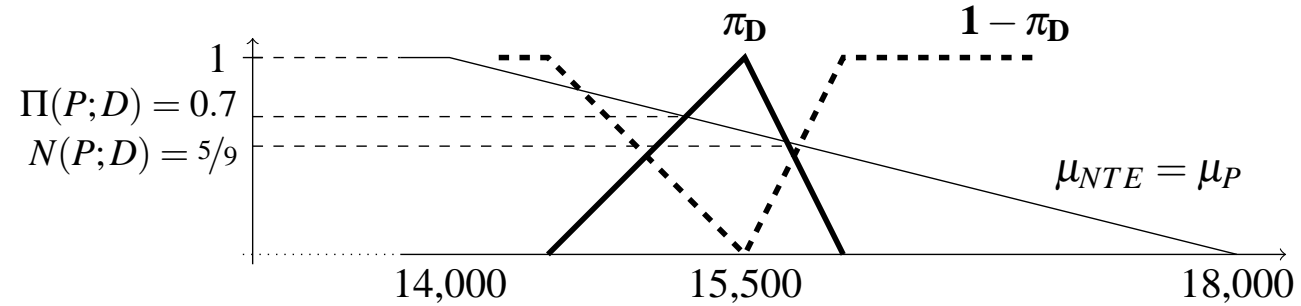

FIGURE 2. Evaluation of a fuzzy preference with uncertain data.

of $P$ given uncertainty $D$ is summarised by the following lower and upper values [20, 19]:

$$
\begin{gathered}
\Pi(P ; D)=\sup _{x \in \mathscr{X}} \min \left(\mu_{P}(x), \pi_{D}(x)\right), \\
N(P ; D)=\inf _{x \in \mathscr{X}} \max \left(\mu_{P}(x), 1-\pi_{D}(x)\right) .
\end{gathered}
$$

In the following, we will speak of evaluations of a fuzzy preference when talking about the interval $[N(P ; D), \Pi(P ; D)]$.

Example 2. Consider the preference of Example1, and a car for which the price is known to belong to the interval $[14,500 ; 16,000]$, with 15,500 the most likely value. Figure 2 illustrates both the preference and the knowledge about the price. From this information, we have (using Eq. (1D) that

$$
\Pi(P ; D)=0.7 \quad \text { and } \quad N(P ; D)=5 / 9
$$

2.2. Notations and problem. The problem we consider is as follows: we assume that we have a database consisting in a set $\mathscr{T}$ of $T$ objects $o_{t}$, $t=1, \ldots, T$, with each object taking its values on the Cartesian product $\times_{i=1}^{N} \mathscr{X}_{i}$ of $N$ domains $\mathscr{X}_{1}, \ldots, \mathscr{X}_{N}$. An object $o_{t}$ is here described by a set of $N$ possibility distributions $\pi_{t}^{i}, i=1, \ldots, N$, where $\pi_{t}^{i}: \mathscr{X}_{i} \rightarrow[0,1]$ is the possibility distribution describing our knowledge about the value of the $i^{t h}$ attribute of object $t$.

We assume that the user provides the following information:

- a set $\mathscr{C}=\left\{C_{1}^{i_{1}}, \ldots, C_{N_{c}}^{i_{N_{c}}}\right\}$ of $N_{c}$ constraints $\left(N_{c} \leq N\right)$ to be satisfied by the retrieved objects, where $C_{j}^{i_{j}}: \mathscr{X}_{i_{j}} \rightarrow[0,1]$ is a normalised fuzzy set defined on the attribute $i_{j}\left(1 \leq i_{j} \leq N\right)$.

- a set $\mathscr{W}=\left\{W_{1}^{i_{1}}, \ldots, W_{N_{w}}^{i_{N_{w}}}\right\}$ of $N_{w}$ wishes $\left(N_{w} \leq N\right)$ that the retrieved objects should satisfy if possible, where $W_{j}^{i_{j}}: \mathscr{X}_{i_{j}} \rightarrow[0,1]$ is a normalised fuzzy set defined on the attribute $i_{j}\left(1 \leq i_{j} \leq N\right)$. 
- complete (pre-)orderings $\leq_{c}$ and $\leq_{w}$ between the constraints to be satisfied and between the wishes, respectively. These (pre)-orderings take account of the fact that some constraints may be considered as more important to satisfy than others (and similarly for wishes). In the sequel, we denote by $\mathscr{C}_{(i)}$ (resp. $\left.\mathscr{W}_{(i)}\right)$ the constraints (resp. the wishes) that have rank $i$ w.r.t. to the (pre-)ordering ${ }^{1} \leq_{c}$ (resp. $\leq_{w}$ ). We denote by $\left|\leq_{c}\right|$ and $\left|\leq_{w}\right|$ the total number of ranks induced by the two orderings.

Note that constraints and wishes may well be defined on the same attribute. For example, having an acceptable price may be considered as a constraint, but since a lower price (all other things being equal) is always preferable, lowering the price may become a wish for prices lower than completely satisfying prices (in Example 1, one can define a wish that would start from 14,000 \$).

Now, the problem we consider here is how to retrieve from a set $\mathscr{T}$ of objects, those that primarily satisfy the constraints, and among these latter ones, those that fulfil the most wishes. Of course, the querying approach has to take account of the bipolar nature of the information, of the possible imprecision in the data, and of the users preferences among constraints and wishes.

We also require that an answer is returned for every query, even if no object is found that satisfies the specified constraints. The reason is that the user may be ready to relax some of his/her constraints, but is often unaware of the constraints that would have to be relaxed in order to have a non-empty answer. Hence, it may be helpful to provide those objects that are close to satisfying the constraints.

\subsection{From bipolar querying with imprecise data to answer ordering.} As underlined by [2], when bipolar information concerns preferences, satisfying constraints should be a primary aim, while satisfying wishes remains secondary. To do this, a solution is to first retain all the objects that may satisfy the constraints, order them w.r.t. the degree to which they satisfy these constraints, and then refine this order by using degrees to which objects satisfy those wishes. If the user has specified preferences between constraints (resp. between wishes) $\sqrt{2}$, we also provide a means to take these preferences into account.

\footnotetext{
${ }^{1}$ Note that since $\leq_{c}$ and $\leq_{w}$ are complete pre-orderings, each constraint/wish has a well-defined rank.

${ }^{2}$ No preferences means here that all constraints (or wishes) have the same rank, i.e., are of equal importance.
} 
We propose, for constraints $\mathscr{C}_{(i)}$ of rank $i$, to summarise the way an object $o_{t}$ satisfies these constraints by an aggregated interval $\left[N_{t}^{(i)}, \Pi_{t}^{(i)}\right]_{c}$ given by the following formula:

$$
N_{t}^{(i)}=\top_{C_{k}^{j_{k} \in \mathscr{C}_{(i)}}} N\left(C_{k}^{j_{k}} ; \pi_{t}^{j_{k}}\right), \quad \text { and } \quad \Pi_{t}^{(i)}=\top_{C_{k}^{j_{k}} \in \mathscr{C}_{(i)}} \Pi\left(C_{k}^{j_{k}} ; \pi_{t}^{j_{k}}\right),
$$

with $N\left(C_{k}^{j_{k}} ; \pi_{t}^{j_{k}}\right), \Pi\left(C_{k}^{j_{k}} ; \pi_{t}^{j_{k}}\right)$ given by Eq. (1), and T a t-norm [3 [25]. Tnorms are conjunctive aggregation operators and are chosen here for the reason that ALL constraints have to be satisfied simultaneously. Here, we take $T=\min$, the minimum operator.

Similarly, we build, for each $\mathscr{W}_{(i)}$ and object $o_{t}$ satisfying the constraints, the interval $\left[N_{t}^{(i)}, \Pi_{t}^{(i)}\right]_{w}$, such that

$$
N_{t}^{(i)}=\oplus_{W_{k}^{j_{k} \in \mathscr{W}_{(i)}}} N\left(W_{k}^{j_{k}} ; \pi_{t}^{j_{k}}\right), \quad \text { and } \quad \Pi_{t}^{(i)}=\oplus_{W_{k}^{j_{k}} \in \mathscr{W}_{(i)}} \Pi\left(W_{k}^{j_{k}} ; \pi_{t}^{j_{k}}\right),
$$

where $\oplus$ is an aggregation operator that can be a t-norm, an averaging operator such as an OWA [32] operator or a t-conorm, depending the behaviour we want to adopt w.r.t. the satisfaction of wishes. Indeed, since satisfying wishes is not compulsory, we can adopt different attitudes [2]. For instance, using a t-conorm means that we are satisfied as soon as one wish is fulfilled, while using a t-norm means that we still want all the wishes to be satisfied to increase our overall satisfaction. In this paper, we consider the latter case, and will consider $\oplus=$ min.

It is then necessary to order objects that could satisfy the constraints and some wishes, according to the previous evaluations. To do so, we will use a lexicographic order and a dominance relation $\leq_{\left[N^{(i)}, \Pi^{(i)}\right]}$ between objects such that, for two interval evaluations $\left[N_{t}^{(i)}, \Pi_{t}^{(i)}\right],\left[N_{t^{\prime}}^{(i)}, \Pi_{t^{\prime}}^{(i)}\right]$ related to objects $o_{t}$ and $o_{t^{\prime}}$ and to a group of constraints $\mathscr{C}_{(i)}$ or a group of wishes $\mathscr{W}_{(i)}$, $o_{t} \leq_{\left[N^{(i)}, \Pi^{(i)}\right]} o_{t^{\prime}}$ if $N_{t}^{(i)} \leq N_{t^{\prime}}^{(i)}$ and $\Pi_{t}^{(i)} \leq \Pi_{t^{\prime}}^{(i)}$ (with $o_{t}<_{\left[N^{(i)}, \Pi^{(i)}\right]} o_{t^{\prime}}$ if at least one inequality is strict). That is, an object $o_{t^{\prime}}$ dominates another one $o_{t}$ if its satisfaction bounds are pair-wise higher than the satisfaction bounds of $o_{t}$. The lexicographic order is then used to take account of the difference between negative and positive preferences and of the orderings $\leq_{c}$ and $\leq_{w}$ (i.e. objects are first ordered using constraints of rank one, then two, ...).

Note that, although $\leq_{\left[N^{(i)}, \Pi^{(i)}\right]}$ is a partial order, we will induce from it a complete (pre-)order that refines $\leq_{\left[N^{(i)}, \Pi^{(i)}\right]}$, for the reason that users are more at ease with complete orderings. However, we will use the fact that

\footnotetext{
${ }^{3} \mathrm{~A}$ T-norm $\top:[0,1]^{2} \rightarrow[0,1]$ is an associative, commutative operator that has one for neutral element and zero for absorbing element.
} 
$\leq_{\left[N^{(i)}, \Pi^{(i)}\right]}$ is a partial order to differentiate negative from positive preferences. The ordering procedure consists in building iteratively an ordered partition $\left\{\mathscr{T}_{0}, \ldots, \mathscr{T}_{M}\right\}$ of $\mathscr{T}$. Rejected objects that do not satisfy all constraints are put in $\mathscr{T}_{0}$, while objects in $\mathscr{T}_{M}$ can be considered as the most satisfactory.

In a preliminary step, Algorithm 1 describes how the objects of $\mathscr{T}$ that do not satisfy at all some constraints, are rejected. If all objects are rejected, the user is warned and a guaranteed answer method, described in Sec. 2.4 is then used to return possible interesting answers to the user.

Algorithm 1: Determination of $\mathscr{T}_{0}$, the set of rejected objects which will not belong to the query result

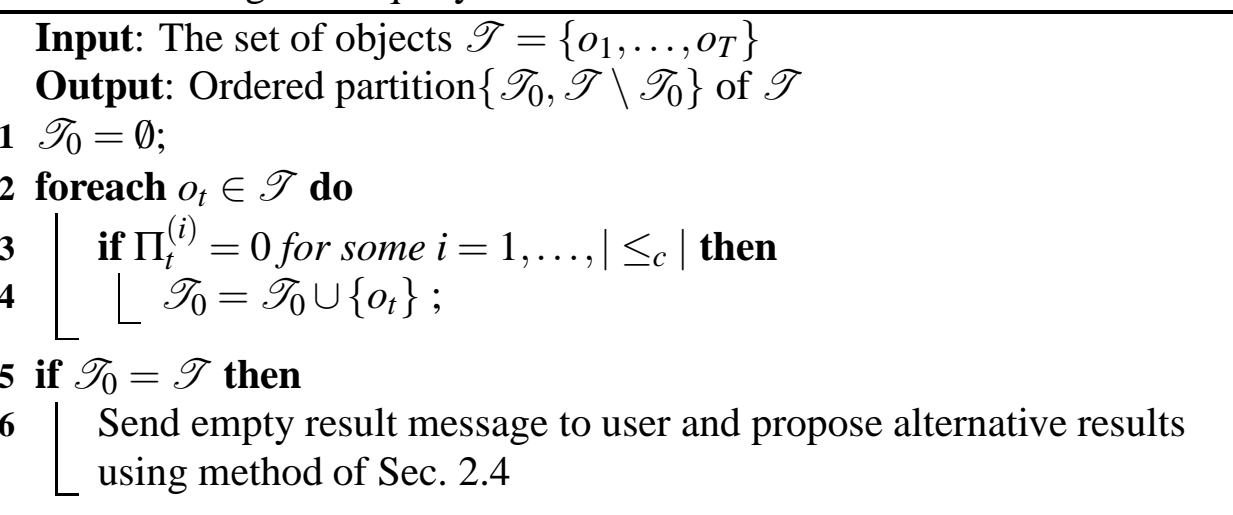

Algorithm 2] describes how results are ordered within a subset of $\mathscr{T} \backslash \mathscr{T}$ (called $\mathscr{T}^{\prime}$ ), according to constraints of a given rank. The whole procedure consists in building a partition of $\mathscr{T} \backslash \mathscr{T}_{0}$. The partition is refined iteratively by applying, at every rank $i\left(i \in\left[1,\left|\leq_{c}\right|\right]\right)$, Algorithm 2 within each equivalence class of objects obtained at the previous rank $i-1$. When $i=1$, the unique initial equivalence class $\mathscr{T}^{\prime}$ is $\mathscr{T} \backslash \mathscr{T}_{0}$. In every run of Algorithm 2, equivalence classes $\left\{\mathscr{T}_{1}^{\prime}, \ldots, \mathscr{T}_{n}^{\prime}\right\}$ are incrementally built, starting from the worst $\left(\mathscr{T}_{1}^{\prime}\right)$ and ending with the best $\left(\mathscr{T}_{n}^{\prime}\right)$. At each step, the objects included and then suppressed from $\mathscr{T}^{\prime}$ are those objects that do not dominate other objects (line 4), in the sense of $\leq_{\left[N^{(i)}, \Pi^{(i)}\right]}$. This means that objects with imprecise evaluations (i.e., $\left[N_{t}^{(i)}, \Pi_{t}^{(i)}\right]$ with larger width) will be in lower classes, along with objects having low evaluations (i.e., low $\left.\Pi_{t}^{(i)}\right)$. This corresponds to a pessimistic attitude towards imprecision, since imprecise evaluations are associated to poorly satisfying objects. Such an attitude is coherent with negative preferences, as the possibility of not satisfying a constraint is penalised. 
0344

0345

0346

0347

0348

0349

0350

0351

0352

0353

0354

0355

0356

0357

0358

0359

0360

0361

0362

0363

0364

0365

0366

0367

0368

0369

0370

0371

0372

0373

0374

0375

0376

0377

0378

0379

0380

0381

0382

0383

0384

0385

0386

0387

0388

0389

0390

0391

0392

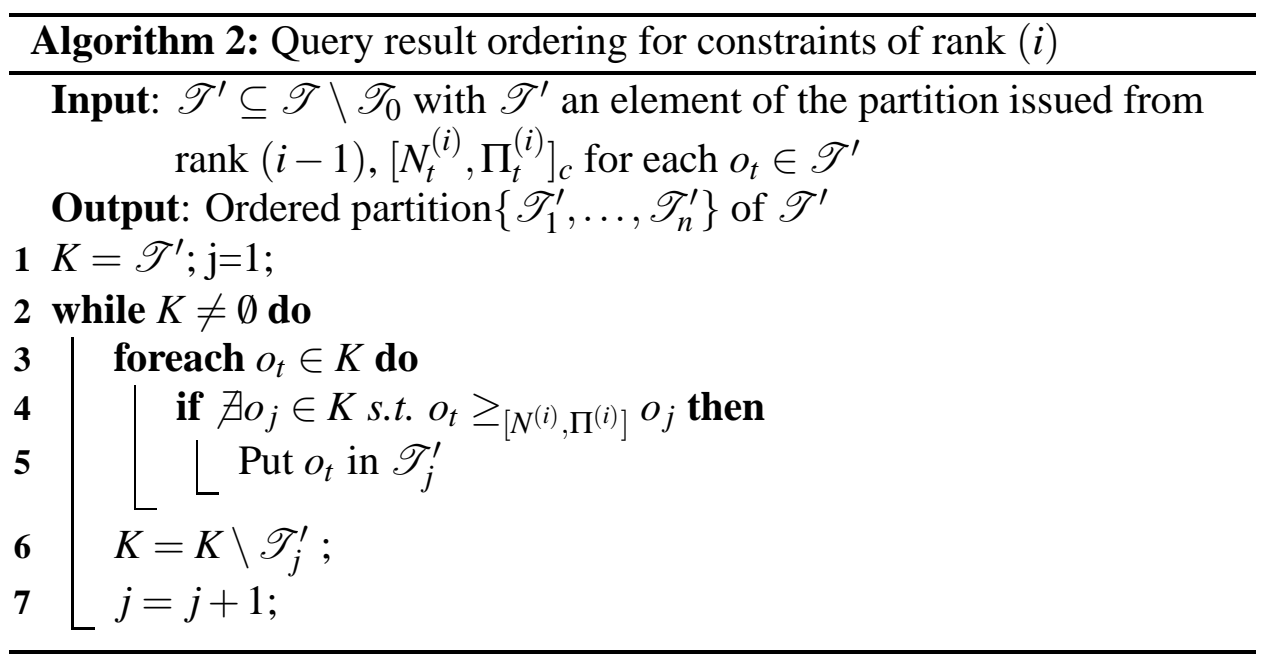

After having applied Algorithm 1 once and Algorithm $2\left|\leq_{c}\right|$ times, the complete pre-order is further refined according to wishes by using Algorithm 3. There are two main differences with Algorithm 1 and Algorithm 2 . First, no objects are rejected, as we are dealing with positive preferences (satisfying them is not compulsory). Second, we start here from the best equivalence class and finish with the worst 4 , and at each step the objects included and then suppressed from $\mathscr{T}^{\prime}$ are those objects that are not dominated by other objects (line 7), in the sense of $\leq_{\left[N^{(i)}, \Pi^{(i)}\right]}$. Contrarily to Algorithm 2, objects with imprecise evaluations will be in the upper classes. This corresponds to an optimistic attitude towards imprecision, which is coherent with positive preferences, as it promotes the possibility of satisfying more wishes. Also notes that the problem of inconsistency raised, for example, in [2], does not happen here, since constraints and wishes are treated separately and lexicographically.

By fully acknowledging the knowledge imprecision through the use of the partial order $\leq_{\left[N^{(i)}, \Pi^{(i)}\right]}$ (that considers both end-points of intervals $\left[N^{(i)}, \Pi^{(i)}\right]$ ), Algorithms 2 and 3 allow to make a clear distinction in the treatment of negative and positive aspects of bipolar preferences. However, a possible drawback, for huge databases, is the complexity that represent the use of these algorithms. Indeed, each run of Algorithms 2 and 3 requires to compare each object with all the other objects of a same equivalence class. If $n$ objects have to be ordered, then in the worst case $\left(\left|\leq_{c}\right|+\left|\leq_{w}\right|\right) n^{2}$ comparisons are performed, assuming that no object strictly dominates another for any rank of constraints or wishes. In the best case, that is when objects are completely ordered after a first run, $n^{2}$ comparisons have to be achieved. It

\footnotetext{
${ }^{4}$ The shift loop (Lines 3-5) is there to keep the same indexing of subsets $\mathscr{T}_{j}$
} 


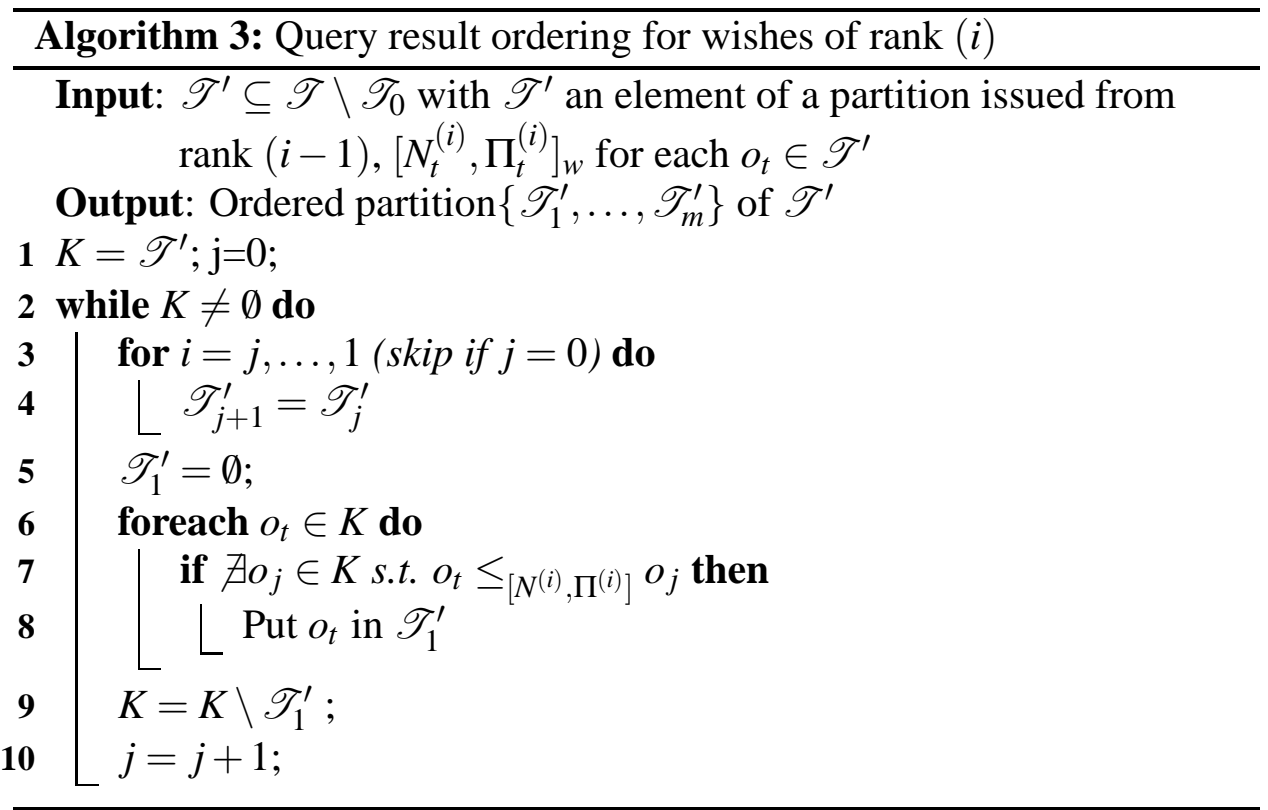

\begin{tabular}{cccc} 
& {$\left[N_{t}^{(1)}, \Pi_{t}^{(1)}\right]_{c}$} & {$\left[N_{t}^{(2)}, \Pi_{t}^{(2)}\right]_{c}$} & {$\left[N_{t}^{(1)}, \Pi_{t}^{(1)}\right]_{w}$} \\
\hline$o_{1}$ & {$[0.1,0.4]$} & {$[0.8,1]$} & {$[1,1]$} \\
$o_{2}$ & {$[0.5,0.8]$} & {$[0.5,0.6]$} & {$[0.6,0.9]$} \\
$o_{3}$ & {$[0.3,1]$} & {$[0.4,0.8]$} & {$[0.2,0.5]$} \\
$o_{4}$ & {$[0.8,1]$} & {$[0,0]$} & {$[0.5,0.7]$} \\
$o_{5}$ & {$[1,1]$} & {$[0.2,0.4]$} & {$[0,0]$} \\
$o_{6}$ & {$[0,1]$} & {$[0.6,0.9]$} & {$[0.3,0.7]$}
\end{tabular}

TABLE 1. Example 3 evaluations for constraints and wishes.

must be noticed that $n$ is reduced to $\left|\mathscr{T} \backslash \mathscr{T}_{0}\right|$ thanks to Algorithm 1, Such complexities are quite acceptable for most databases, but could be problematic for databases counting billions of objects. In such a case, one can then use other propositions presenting a lower complexity where object ordering is solely based on one of the two numbers $N^{(i)}$ or $\Pi^{(i)}$ [20]. Still, by using orderings based on single numbers, one does not take full account of the imprecision in $\left[N^{(i)}, \Pi^{(i)}\right]$, and loose some of the information contained in the interval.

Example 3. Let us consider a set $\mathscr{T}$ of six objects $o_{1}, \ldots, o_{6}$, two ranks of constraints and only one rank of wish. The intervals $\left[N_{t}^{(i)}, \Pi_{t}^{(i)}\right]_{c}(i=\{1,2\})$ and $\left[N_{t}^{(1)}, \Pi_{t}^{(1)}\right]_{w}$ are summarized in table 1 . 
The run of Algorithm 1 gives $\mathscr{T}_{0}=\left\{o_{4}\right\} . o_{4}$ is the only rejected object, because $\Pi_{4}^{(2)}=0$, even if it satisfies rank 1 constraints necessarily to a high degree. After a first run of Algorithm 2, we obtain the following partition:

$$
\mathscr{T}_{0}=\left\{o_{4}\right\}<\mathscr{T}_{1}=\left\{o_{1}, o_{6}\right\}<\mathscr{T}_{2}=\left\{o_{2}, o_{3}\right\}<\mathscr{T}_{3}=\left\{o_{5}\right\} .
$$

All elements potentially satisfy constraints in $\mathscr{C}_{(1)}$ (although $o_{6}$ does not necessarily satisfy it). Note that $o_{6}$, for which information is fully imprecise, is at the end of the ordering (while it would have been at the front if we used Algorithm 3). Since there is two rank of constraints, a second run of Algorithm 2 gives

$$
\mathscr{T}_{0}=\left\{o_{4}\right\}<\mathscr{T}_{1}=\left\{o_{6}\right\}<\mathscr{T}_{2}=\left\{o_{1}\right\}<\mathscr{T}_{3}=\left\{o_{2}, o_{3}\right\}<\mathscr{T}_{4}=\left\{o_{5}\right\} .
$$

This second run has allowed to refine the ordering between $o_{1}$ and $o_{6}$. Also note that the bad scores of $o_{5}$ w.r.t. constraints of rank 2 does not change its order, due to the constraints preferences and the use of a lexicographic order. Finally, a run of Algorithm 3 gives

$\mathscr{T}_{0}=\left\{o_{4}\right\}<\mathscr{T}_{1}=\left\{o_{6}\right\}<\mathscr{T}_{2}=\left\{o_{1}\right\}<\mathscr{T}_{3}=\left\{o_{3}\right\}<\mathscr{T}_{4}=\left\{o_{2}\right\}<\mathscr{T}_{5}=\left\{o_{5}\right\}$.

Note that $o_{5}$ is not rejected, for the reason that satisfying wishes is not a requirement.

2.4. Guaranteeing some answers. In Example 3 , the set $\mathscr{T}_{0}$ of rejected objects did not contain all of them (i.e., $\mathscr{T} \neq \mathscr{T}$ ), and there were positive answers to the query. However, it may happen that a query has no satisfying answer. This may be the result of a small number of available objects (hence there are less chances of satisfying all constraints) or of the specification of unrealistic or narrow constraints. In such a case, it may be difficult for a user to know why its query has received no answers, and which constraints should be relaxed to get some.

This is why, in this section, we propose a method guaranteeing the presence of some answers, even when $\mathscr{T}_{0}=\mathscr{T}$. The principle is quite simple: after having sent a message to the user (see Algorithm 11), it consists in returning the set $\mathscr{T}$ of objects (or a subset of $\mathscr{T}$ ), ordered w.r.t. to the gap between these objects' attribute values and values they should have in order not to be rejected.

First, recall that the support of a fuzzy set $F$ defined on $\mathscr{X}$, denoted by $S(F)$, is the set such that $S(F)=\{x \in \mathscr{X} \mid F(x)>0\}$.

We assume that, for every attribute $j$, a (normalized) distance $d_{j} \in[0,1]$ on $\mathscr{X}_{j}$ is defined. Consider the set of supports $\left\{S\left(C_{1}^{i_{1}}\right), \ldots, S\left(C_{N_{c}}^{i_{N_{c}}}\right)\right\}$ obtained from the set $\mathscr{C}$ of constraints. For an object $o_{t}$ and an attribute $j$, the 
distance between $\pi_{t}^{j}$ and a constraint $C^{j}$ is defined as the following value:

$$
D_{j}\left(\pi_{t}^{j}, C^{j}\right)=\min _{x \in S\left(\pi_{t}^{j}\right), y \in S\left(C^{j}\right)}\left(\left|d_{j}(x, y)\right|\right) .
$$

This distance can be seen as the minimal modification a constraint $C^{j}$ would have to undergo so that object $o_{t}$ satisfies it. Now, we define the global distance of an object $o_{t}$ w.r.t. constraints in $\mathscr{C}$ as a function $\delta$ having value in $\left[0, N_{c}\right]$ and such that:

$$
\delta\left(o_{t}, \mathscr{C}\right)=\sum_{k=1}^{N_{c}} w_{(k)} D_{i_{k}}\left(\pi_{t}^{i_{k}}, C_{k}^{i_{k}}\right)
$$

with $(k)$ the rank of $C_{k}^{i_{k}}$ according to $\leq_{c}$ and $w_{(k)}$ a weight reflecting the constraint importance. A sum is used to translate the fact that an almost acceptable object should be (in average) close to all constraints. Objects in $\mathscr{T}_{0}$ can then be ordered according to distance $\delta$. We propose the following formula for the weights $w_{(k)}$ for any $k \in 1, \ldots,\left|\leq_{c}\right|$ :

$$
w_{(k)}=\frac{\left|\leq_{c}\right|+1-k}{\left|\leq_{c}\right|}
$$

that is, weights are in the interval $[0,1]$ and are linearly ordered. A possible inconvenient when using an averaging operator is that objects violating very strongly only one constraint can have the same evaluation as objects weakly violating many constraints. Two possible solutions to this problem are to provide the user with a list of the violated constraint, or to use some kind of veto level (as in [11]) to penalise objects strongly violating some constraints (this latter option meaning that the user must supply such veto levels).

Example 4. Let us consider a set $\mathscr{T}=\left\{o_{1}, \ldots, o_{3}\right\}$ of three objects with two constraints expressed in the query. The intervals $\left[N_{t}^{i}, \Pi_{t}^{i}\right]_{c}(i=\{1,2\})$ and the minimal distances to the constraints $D_{i}\left(\pi_{t}^{i}, C^{i}\right)(i=\{1,2\})$ are given in Table 2. In this example, there is no positive answer to the query because, at least one of the constraints is not satisfied (i.e., $\mathscr{T}=\mathscr{T}_{0}$ ) for each object. According to distance $\delta$ and to the weights $w_{(1)}=1, w_{(2)}=1 / 2$, objects are ranked in the following order: $o_{1}, o_{3}, o_{2}$.

There exists other methods to obtain answers from empty queries. They mainly consist of relaxing the initial query into a less constraining query using, for example, fuzzy membership function transformations [5] or casebased reasoning techniques [4]. These approaches are arguably more subtle and efficient but necessitate to specify many parameters and to apply (possibly many times) new queries, and are therefore computationally more demanding. Our aim here was to propose a simple but meaningful and efficient technique, that would not require additional parameters. 
0540

0541

0542

0543

0544

0545

0546

0547

0548

0549

0550

0551

0552

0553

0554

0555

0556

0557

0558

0559

0560

0561

0562

0563

0564

0565

0566

0567

0568

0569

0570

0571

0572

0573

0574

0575

0576

0577

0578

0579

0580

0581

0582

0583

0584

0585

0586

0587

0588

\begin{tabular}{cccccc} 
& {$\left[N_{t}^{1}, \Pi_{t}^{1}\right]_{c}$} & {$\left[N_{t}^{2}, \Pi_{t}^{2}\right]_{c}$} & $D_{1}\left(\pi_{t}^{1}, C^{1}\right)$ & $D_{2}\left(\pi_{t}^{2}, C^{2}\right)$ & $\delta\left(o_{t}, \mathscr{C}\right)$ \\
\hline$o_{1}$ & {$[0.0,0.0]$} & {$[0.8,1]$} & 0.1 & 0 & 0.1 \\
$o_{2}$ & {$[0.0,0.0]$} & {$[0.0,0.0]$} & 0.6 & 0.5 & 0.85 \\
$o_{3}$ & {$[0.3,0]$} & {$[0.0,0.0]$} & 0 & 0.7 & 0.35
\end{tabular}

TABLE 2. Example 4 evaluations for two constraints and the minimal distances to the constraints.

\section{A NEW DECISION SUPPORT SYSTEM FOR FOOD PACKAGING DESIGN}

In this section, we present a new decision support system (DSS) for fresh fruits and vegetables packaging design in which the flexible bipolar querying approach plays a central role. To the best of our knowledge, only one DSS for fresh fruits and vegetables packaging already exists (see [26]), but it does not take into account the criteria which permit to ensure a sustainable design (a critical issue in food science). Such a sustainable design must satisfy, at least, three kinds of criteria: economical, environmental and societal. An example of economical aspect may be the cost of the packaging material. Concerning environmental aspects, the biodegradability of the package or the way to optimize the preservation of fresh fruits and vegetables at ambient temperature in order to decrease the use of the cold chain (which is energy-greedy) are important criteria. Societal aspects may concern the fact that consumers may reject the use of some additives or of nano-technology in the packaging material because of the unknown consequences on their health, or more simply they may prefer transparent rather than opaque packaging.

In our DSS, starting from a given fruit or vegetable, the user specifies its needs in terms of several criteria (e.g., conservation temperature, transparency, material cost, ... ) in order to determine a list of packagings. Those packagings are ordered according to their degree of satisfaction of the criteria. The bipolar approach gives the user the possibility to specify in a flexible way what criteria must be considered as constraints and what other criteria will be used to refine the ranking of the packages which satisfy the constraints. Starting from the users' specifications, a flexible bipolar query is executed against a database containing information about packaging materials. This information has been collected from different sources which may be technical descriptions of commercial packaging materials or data extracted from scientific publications concerning new packaging materials. This information may be imprecise, due to the variability of engineered packaging and the biological variability of vegetables. The bipolar approach proposed in this paper takes into account this imprecision. 
In Section 3.1, we present the global architecture of the DSS. A use case concerning endive packaging will be presented in Section 3.2 .

3.1. Decision support system architecture. Starting from the name of the vegetable/fruit of interest specified by the user, the system scans in a first step the vegetable/fruit database in order to retrieve the $\mathrm{O} 2$ respiration rate (and associated parameters) of the studied vegetable/fruit. In a second step, the optimal $\mathrm{O} 2$ permeance 5 of the targeted packaging is computed thanks to a model of gas exchanges inside the package called PassiveMap (see [15] for more details about the model). In the third step, using the targeted optimal $\mathrm{O} 2$ permeance and the other user's requirements about criteria of various types (economical, environmental or societal), a query is executed against the packaging database using the flexible bipolar querying engine, which is the central part of the DSS. A list of packaging materials ordered according to the method presented in the previous sections is finally presented to the user. A precise use case presented in the next section focuses on the DSS flexible bipolar querying engine.

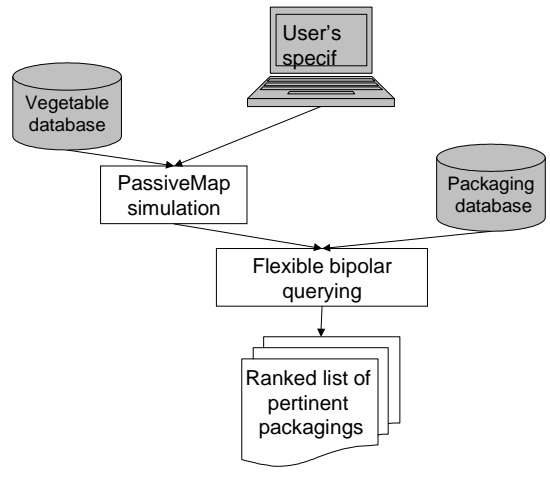

FIGURE 3. Global architecture of the DSS

3.2. Endive packaging use case. In this section, we present a use case of the DSS concerning the choice of a package for endive. The user has to specify a set of parameters needed by the DSS to determine the optimal $\mathrm{O}_{2}$ permeance of the targeted packaging: the mass of the vegetable (500 grams), the surface, the volume and the thickness of the targeted packaging (respectively $0.14 \mathrm{~m}^{2}, 0.002 \mathrm{~m}^{3}$ and $5 \mathrm{e}-5 \mathrm{~m}$ ), the shelf life of the vegetable (7 days) and the storage temperature $\left(20{ }^{\circ} \mathrm{C}\right)$. Using the $\mathrm{O}_{2}$ respiration rate (and associated parameters) retrieved from the vegetable database, an optimal $\mathrm{O}_{2}$ permeance of $3.65 \mathrm{E}-11 \mathrm{~mol} \cdot \mathrm{m}^{-2} \cdot \mathrm{s}^{-1} \cdot \mathrm{Pa}^{-1}$ is computed. The

\footnotetext{
${ }^{5} \mathrm{~A}$ measure of the ability of a package to conduct gas fluxes.
} 
0638

0639

0640

0641

0642

0643

0644

0645

0646

0647

0648

0649

0650

0651

0652

0653

0654

0655

0656

0657

0658

0659

0660

0661

0662

0663

0664

0665

0666

0667

0668

0669

0670

0671

0672

0673

0674

0675

0676

0677

0678

0679

0680

0681

0682

0683

0684

0685

0686

\begin{tabular}{ccccc}
$o_{\text {id }}$ & PackagingType & $\begin{array}{c}\text { Permeance } \min \\
\left(\text { mol. }^{-2} \cdot s^{-1} \cdot \mathrm{Pa}^{-1}\right)\end{array}$ & $\begin{array}{c}\text { Permeance } \\
\left(\mathrm{mol}_{\mathrm{max}} \mathrm{m}^{-2} \cdot \mathrm{s}^{-1} \cdot \mathrm{Pa}^{-1}\right)\end{array}$ & $\begin{array}{c}\text { Temperature } \\
\left({ }^{\circ} \mathrm{C}\right)\end{array}$ \\
\hline$o_{1}$ & Polyolefin & $1,29 E-13$ & $1,29 E-13$ & 23 \\
$o_{2}$ & Polyolefin & $4,05 E-11$ & $4,05 E-11$ & 23 \\
$o_{3}$ & Cellophane & $1,55 E-14$ & $1,55 E-14$ & 23 \\
$o_{4}$ & Polyolefin & $1,96 E-11$ & $2,39 E-11$ & 20 \\
$o_{5}$ & Cellulose & $1,55 E-14$ & $1,55 E-14$ & 23 \\
$o_{6}$ & Polyester & $4,46 E-12$ & $4,46 E-12$ & 23 \\
$o_{7}$ & Polyolefin & $1,50 E-11$ & $1,50 E-11$ & 23 \\
$o_{8}$ & Polyester & $1,55 E-13$ & $1,55 E-13$ & 23 \\
$o_{9}$ & Polystyrene & $1,03 E-12$ & $1,03 E-12$ & 23 \\
$o_{10}$ & Polyester & $6,23 E-12$ & $6,23 E-12$ & 23 \\
$o_{11}$ & Wheatgluten & $1,55 E-11$ & $1,67 E-11$ & 25 \\
$o_{12}$ & PolyVinylChloride & $7,47 E-11$ & $7,47 E-11$ & 25
\end{tabular}

TABLE 3. Permeance at a given temperature for a excerpt of the packaging database

optimal permeance and the temperature will be considered as criteria to scan the package database.

We consider in this use case that the user is also interested in two other criteria: the biodegradability and the transparency of the package. An excerpt of the packaging database content is presented in Tables 3 and 4 and will be used to illustrate the flexible bipolar querying process. Note that imprecise data are here reduced to degenerated possibility distributions (given by the $\min -\max$ permeance span), for the reason that there are currently no possibilistic imprecise data in the database.

We will consider two examples of queries expressed by the user (in the current case, they were given by one of the co-authors, V. Guillard). In the first one, the user specifies one constraint and two wishes. The user first requires the package to be transparent in order to be accepted by the consumer who wants to see endive through the package. It will be expressed as the first and unique constraint. Concerning his/her wishes, the user would like to maximize the shelf life of the product at an ambient temperature (and consequently to select a packaging whose oxygen permeance is close to the optimal one). It will be expressed as the wishes, here of equal rank.

In the second query, the user specifies three constraints and one wish. To design a sustainable package, the user expresses that the packaging must be biodegradable as a first constraint (rank one) and must also maximize the shelf life of the product at an ambient temperature as a second constraint (i.e. constraints of rank two on the temperature and oxygen permeance). 
FLEXIBLE BIPOLAR QUERYING OF IMPRECISE DATA

0687

0688

0689

0690

0691

0692

0693

0694

0695

0696

0697

0698

0699

0700

0701

0702

0703

0704

0705

0706

0707

0708

0709

0710

0711

0712

0713

0714

0715

0716

0717

0718

0719

0720

0721

0722

0723

0724

0725

0726

0727

0728

0729

0730

0731

0732

0733

0734

0735

\begin{tabular}{cccc}
$o_{i d}$ & PackagingType & Transparency & Biodegradability \\
\hline$o_{1}$ & Polyolefin & transparent & no \\
$o_{2}$ & Polyolefin & transparent & no \\
$o_{3}$ & Cellophane & transparent & yes \\
$o_{4}$ & Polyolefin & transparent & no \\
$o_{5}$ & Cellulose & transparent & yes \\
$o_{6}$ & Polyester & transparent & yes \\
$o_{7}$ & Polyolefin & transparent & no \\
$o_{8}$ & Polyester & translucent & yes \\
$o_{9}$ & Polystyrene & translucent & no \\
$o_{10}$ & Polyester & translucent & yes \\
$o_{11}$ & Wheatgluten & translucent & yes \\
$o_{12}$ & PolyVinylChloride & transparent & no
\end{tabular}

TABLE 4. Transparency and biodegradability for the same excerpt of the packaging database

Then, the user expresses as its only wish that the packaging should be transparent for acceptability of this new packaging material by the consumer.

As already said in Section 2.1, user preferences for each criterion is expressed as a fuzzy set used as a general formalism which permits to represent fuzzy, interval or crisp values. Concerning the permeance criterion, $60 \%$ of variation is authorized around the optimal value computed by the PassiveMap subsystem, with decreasing degrees of preferences. For the temperature, a total variation of $100 \%$ is authorised, with no preference for the different values. The fuzzy sets associated with the permeance and temperature preferences are presented in Figure 4
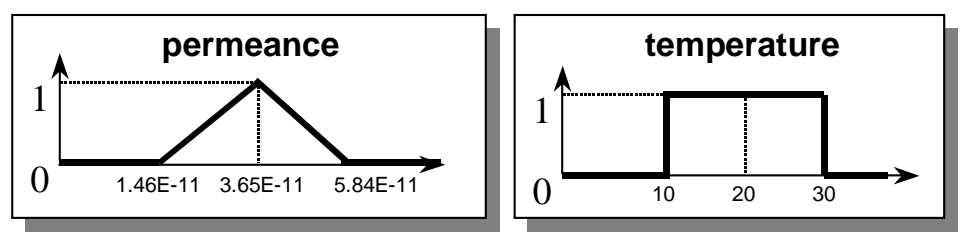

FIGURE 4. Preferences for permeance and temperature

The fuzzy set associated with the transparency (resp. biodegradability) criterion is: Pre $f_{\text {transparency }}=\{($ transparent,1), (translucent,0), (opaque,0) $\}$ (resp.

\footnotetext{
${ }^{6}$ Here, we adopt the usual notation $(x, y)$ for specifying fuzzy sets over symbolic variables, where $(x, y)$ means that modality $x$ has membership value $y$.
} 
0736

0737

0738

0739

0740

0741

0742

0743

0744

0745

0746

0747

0748

0749

0750

0751

0752

0753

0754

0755

0756

0757

0758

0759

0760

0761

0762

0763

0764

0765

0766

0767

0768

0769

0770

0771

0772

0773

0774

0775

0776

0777

0778

0779

0780

0781

0782

0783

0784

\begin{tabular}{ccc} 
& {$\left[N_{t}^{(1)}, \Pi_{t}^{(1)}\right]_{c}$} & {$\left[N_{t}^{(1)}, \Pi_{t}^{(1)}\right]_{w}$} \\
\hline$o_{1}$ & {$[1,1]$} & {$[0,0]$} \\
$o_{2}$ & {$[1,1]$} & {$[0,817,0,817]$} \\
$o_{3}$ & {$[1,1]$} & {$[0,0]$} \\
$o_{4}$ & {$[1,1]$} & {$[0,228,0,427]$} \\
$o_{5}$ & {$[1,1]$} & {$[0,0]$} \\
$o_{6}$ & {$[1,1]$} & {$[0,0]$} \\
$o_{7}$ & {$[1,1]$} & {$[0,021,0,021]$} \\
$o_{8}$ & {$[0,0]$} & {$[0,0]$} \\
$o_{9}$ & {$[0,0]$} & {$[0,0]$} \\
$o_{10}$ & {$[0,0]$} & {$[0,0]$} \\
$o_{11}$ & {$[0,0]$} & {$[0,043,0,098]$} \\
$o_{12}$ & {$[1,1]$} & {$[0,0]$}
\end{tabular}

TABLE 5. Evaluations for the constraint and the wishes of the first query.

Pre $f_{\text {biodegradability }}=\{($ yes, 1$),($ no, 0$\left.)\}\right)$. They correspond to crisp requirements provided by the user, as the concept of graded biodegradability made little sense to the user, while translucency is not graded in our current data.

Using the notations introduced in the section 2.1, the first query is built as follows: $\mathscr{C}_{(1)}=\left\{\right.$ Pref $\left.f_{\text {transparency }}\right\}$ and $\mathscr{W}_{(1)}=\left\{\right.$ Pref $f_{\text {permeance }}$, Pre $\left.f_{\text {temperature }}\right\}$.

Let us consider the set $\mathscr{T}=\left\{o_{1}, \ldots, o_{12}\right\}$ of the twelve packages whose characteristics are given in tables 3 and 4 and whose evaluations for the constraint and wishes of query 1 are given in table 5 (as the two wishes are of the same rank, they have been aggregated in $\left[N_{t}^{(1)}, \Pi_{t}^{(1)}\right]_{w}$ according to Eq. (3)). After the run of Algorithm 1, we obtain $\mathscr{T}_{0}=\left\{o_{8}, o_{9}, o_{10}, o_{11}\right\}$. After the run of Algorithm 2 with $\mathscr{C}_{(1)}$, we obtain the following partition:

$$
\mathscr{T}_{0}=\left\{o_{8}, o_{9}, o_{10}, o_{11}\right\}<\mathscr{T}_{1}=\left\{o_{1}, o_{2}, o_{3}, o_{4}, o_{5}, o_{6}, o_{7}, o_{12}\right\} .
$$

After the run of Algorithm 3 with $\mathscr{W}_{(1)}$, we obtain the following partition:

$$
\begin{gathered}
\mathscr{T}_{0}=\left\{o_{8}, o_{9}, o_{10}, o_{11}\right\}<\mathscr{T}_{1}=\left\{o_{1}, o_{3}, o_{5}, o_{6}, o_{12}\right\}< \\
\mathscr{T}_{2}=\left\{o_{7}\right\}<\mathscr{T}_{3}=\left\{o_{4}\right\}<\mathscr{T}_{4}=\left\{o_{2}\right\} .
\end{gathered}
$$

The second query is built as follows: $\mathscr{C}_{(1)}=\left\{\right.$ Pref $\left._{\text {biodegradability }}\right\}, \mathscr{C}_{(2)}=$ $\left\{\right.$ Pref $_{\text {permeance }}$, Pref $\left.f_{\text {temperature }}\right\}$ and $\mathscr{W}_{(1)}=\left\{\right.$ Pref $\left.f_{\text {transparency }}\right\}$. The first constraint is judged more important than the two others, which are of equal rank. 


\begin{tabular}{cccc} 
& {$\left[N_{t}^{(1)}, \Pi_{t}^{(1)}\right]_{c}$} & {$\left[N_{t}^{(2)}, \Pi_{t}^{(2)}\right]_{c}$} & {$\left[N_{t}^{(1)}, \Pi_{t}^{(1)}\right]_{w}$} \\
\hline$o_{1}$ & {$[0,0]$} & {$[0,0]$} & {$[1,1]$} \\
$o_{2}$ & {$[0,0]$} & {$[0,817,0,817]$} & {$[1,1]$} \\
$o_{3}$ & {$[1,1]$} & {$[0,0]$} & {$[1,1]$} \\
$o_{4}$ & {$[0,0]$} & {$[0,228,0,427]$} & {$[1,1]$} \\
$o_{5}$ & {$[1,1]$} & {$[0,0]$} & {$[1,1]$} \\
$o_{6}$ & {$[1,1]$} & {$[0,0]$} & {$[1,1]$} \\
$o_{7}$ & {$[0,0]$} & {$[0,021,0,021]$} & {$[1,1]$} \\
$o_{8}$ & {$[1,1]$} & {$[0,0]$} & {$[0,0]$} \\
$o_{9}$ & {$[0,0]$} & {$[0,0]$} & {$[0,0]$} \\
$o_{10}$ & {$[1,1]$} & {$[0,0]$} & {$[0,0]$} \\
$o_{11}$ & {$[1,1]$} & {$[0,043,0,098]$} & {$[0,0]$} \\
$o_{12}$ & {$[0,0]$} & {$[0,0]$} & {$[1,1]$}
\end{tabular}

TABLE 6. Evaluations for the constraints and the wish of the second query.

Let us consider the set $\mathscr{T}$ of the twelve packages $o_{1}, \ldots, o_{12}$ whose characteristics are given in tables 3 and 4 and whose evaluations for the constraints and the wish of query 2 are given in table 6 . After the run of Algorithm 1, we obtain $\mathscr{T}_{0}=\left\{o_{1}, o_{2}, o_{3}, o_{4}, o_{5}, o_{6}, o_{7}, o_{8}, o_{9}, o_{10}, o_{12}\right\}$. After the first run of Algorithm 2 with $\mathscr{C}_{(1)}$, we obtain the following partition:

$$
\mathscr{T}_{0}=\left\{o_{1}, o_{2}, o_{3}, o_{4}, o_{5}, o_{6}, o_{7}, o_{8}, o_{9}, o_{10}, o_{12}\right\}<\mathscr{T}_{1}=\left\{o_{11}\right\} .
$$

Since only one object is not rejected, the second run of Algorithm 2 with $\mathscr{C}_{(2)}\left(\left[N_{t}^{(2)}, \Pi_{t}^{(2)}\right]_{c}\right)$ and the run of Algorithm 3 with $\mathscr{W}_{(1)}$ keeps the partition unchanged.

We can see with the result obtained for the second query, from which only one result is retrieved, that the constraints may be very restrictive compared to the content of the database. We consider in the following a third query, derived from query 2 and defined as follows: $\mathscr{C}_{(1)}=\left\{\right.$ Pre $\left._{\text {biodegradability }}\right\}$, $\mathscr{C}_{(2)}=\left\{\right.$ Pref $f_{\text {permeance_restricted }}$, Pref $\left.f_{\text {temperature }}\right\}$ and $\mathscr{W}_{(1)}=\left\{\right.$ Pref $\left.f_{\text {transparency }}\right\}$. In this query, we restrict the variation around the permeance optimal value (3.65E-11 mol. $\mathrm{m}^{-2} \cdot \mathrm{s}^{-1} \cdot \mathrm{Pa}^{-1}$ ) from $60 \%$ to only $50 \%$. Consequently, the support of the restricted fuzzy set Pref permeance_restricted is equal to the interval [1.83E-11, 5.48E-11] mol. $\mathrm{m}^{-2} \cdot \mathrm{s}^{-1} \cdot \mathrm{Pa}^{-1}$. Query 3 being more restrictive than query 2 and considering the permeance value associated with package $o_{11}$ in Table 3, we can see that the result of query 3 is empty.

In this case, our method will provide guaranteed results which are defined as the set of packages ordered w.r.t. to the gap between the packaging attribute values and the values they should have in order not to be rejected. 
0834

0835

0836

0837

0838

0839

0840

0841

0842

0843

0844

0845

0846

0847

0848

0849

0850

0851

0852

0853

0854

0855

0856

0857

0858

0859

0860

0861

0862

0863

0864

0865

0866

0867

0868

0869

0870

0871

0872

0873

0874

0875

0876

0877

0878

0879

0880

0881

0882

\begin{tabular}{cccccc} 
& $D_{1}\left(\pi_{t}^{1}, C^{1}\right)$ & $D_{2}\left(\pi_{t}^{2}, C^{2}\right)$ & $D_{3}\left(\pi_{t}^{3}, C^{3}\right)$ & $\delta\left(o_{t}, \mathscr{C}\right)$ & rank \\
\hline$o_{1}$ & 1 & 0.6828 & 0 & 1,3414 & 10 \\
$o_{2}$ & 1 & 0 & 0 & 1 & 6 \\
$o_{3}$ & 0 & 0.7545 & 0 & 0.3772 & 5 \\
$o_{4}$ & 1 & 0 & 0 & 1 & 6 \\
$o_{5}$ & 0 & 0.7545 & 0 & 0.3772 & 5 \\
$o_{6}$ & 0 & 0.3813 & 0 & 0.1906 & 3 \\
$o_{7}$ & 1 & 0.0813 & 0 & 1,0406 & 7 \\
$o_{8}$ & 0 & 0.6746 & 0 & 0.3373 & 4 \\
$o_{9}$ & 1 & 0.5555 & 0 & 1,2777 & 9 \\
$o_{10}$ & 0 & 0.3205 & 0 & 0.1602 & 2 \\
$o_{11}$ & 0 & 0.0406 & 0 & 0.0203 & 1 \\
$o_{12}$ & 1 & 0.1187 & 0 & 1,0593 & 8
\end{tabular}

TABLE 7. The minimal distances to the constraints of query 3.

As introduced in section 2.4, we need to define for every object attribute $j$, a normalized distance $d_{j} \in[0,1]$ on $\mathscr{X}_{j}$. In the following, we will consider, for the attribute biodegradability defined on $\mathscr{X}_{\text {biodegradability }}=\{y e s$, no $\}$, the distance $d_{1}(x, y)$ with $(x, y) \in \mathscr{X}_{\text {biodegradability }}^{2}$, which is equal to 1 if $x=y$ and equal to 0 else. For the attributes permeance (resp. temperature) defined on $\mathbb{R}$, we consider the distance $d_{2}(x, y)$ (resp. $d_{3}(x, y)$ ) with $(x, y) \in \mathbb{R}^{2}$ and defined as follows:

$$
d_{2}(x, y)=d_{3}(x, y)=1-\frac{1}{1+|x-y|} .
$$

Note that, given the variation of permeance compared to, e.g. temperature, we have applied a log transformation to it before computing the distance.

Let us consider the set $\mathscr{T}$ of the twelve packages $o_{1}, \ldots, o_{12}$ whose characteristics are given in tables 3 and 4 , the normalized distances given above and the supports of the fuzzy sets Pre $f_{\text {biodegradability }}$, Pre $f_{\text {permeance_restricted, }}$, Pre $f_{\text {temperature }}$ being respectively the singleton $\{$ yes $\}$, the interval $[1.83 \mathrm{E}$ 11, 5.48E-11] mol. $\mathrm{m}^{-2} \cdot \mathrm{s}^{-1} \cdot \mathrm{Pa}^{-1}$ and the interval $[10,30]{ }^{\circ} \mathrm{C}$, the minimal distances to the constraints of query 3 and a rank based on the global distance are given in table 7. We can see that packaging $o_{11}$ is at the first rank which is not surprising because it is the only one which satisfies the constraints of query 2 . Hence, in the above case, the user would be able to see that package $o_{11}$ almost answers his/her needs. 


\section{RELATED WORKS}

There exist many works that propose to use fuzzy sets to introduce graded preferences and possibility distributions to handle uncertainty in databases. Our work can be related to these two complementary propositions

Indeed, the fuzzy set framework has been shown to be a sound scientific choice to model flexible queries ([6]). It is a natural way of representing the notion of preference using a gradual scale. In [8], the semantics of a language called SQLf has been proposed to extend the well-known SQL language by introducing fuzzy predicates processed on crisp information. Other approaches have also been proposed to introduce preferences into queries in the database community $([12,[24,[16])$. However, in all these approaches, preferences are of the same nature. It is only recently that the concept of bipolarity and its potential use in flexible queries has been studied [21, 22]. This extended approach discriminates between two types of preferences, one acting as compulsory constraints, the other acting as optional wishes. Several works have recently been proposed in order to extend the relational algebra with this concept of bipolarity $([9,10])$ or to propose a framework to deal with bipolarity in regular relational databases ([31]).

The second proposition is to use possibility distributions (whose formalism is mathematically equivalent to that of fuzzy set) to represent imprecise values ([34]). Several authors have developed this approach in the context of databases $([27,28,7,3,29,13])$.

To the best of our knowledge, the only other works dealing with the concept of bipolarity in flexible querying of databases including imprecise values, outside some research perspectives in [22], is the one of G. De tr et al. [30]. However, they deal with a different aspect of bipolar preferences, as they mainly consider the use of interval-valued fuzzy sets (or similar models) to cope with imprecisely defined preferences, and treat positive and negative preferences in a common framework, rather than considering them separately (as we do here).

\section{CONCLUSION AND PERSPECTIVES}

In this paper, we have introduced a method for querying a database when preferences are bipolar (contains both constraints and wishes) and data are uncertain. We use fuzzy sets and possibility distributions to model preferences and uncertainty, respectively.

Using basic tools to evaluate query satisfaction, we have proposed methods allowing us to (1) consider orderings between constraints or wishes (2) 
(pre-)order the results according to the bipolar preferences, thus presenting a list of equivalence classes to the user and (3) return potential answers when no object satisfy simultaneously all specified constraints.

The proposed approach is currently applied in a real-case problem, and is included in a new support decision tool aiming at designing (optimal) packages for fresh fruits and vegetables.

Concerning the method, perspectives include the handling of more generic kinds of uncertainty models [18, 17] that could be included in the database, as well as methods that would allow to extract automatically information concerning packages from the web, since manually entering this information is time-consuming and can only be done by an expert.

Concerning the support decision tool, we are planing to link it with a version of PassiveMap able to perform uncertainty analysis and to provide interval-valued or possibilistic valued optimal permeance. The uncertainty analysis will also be able to cope with ill-known values of vegetables parameters, using classical uncertainty propagation techniques [1].

\section{REFERENCES}

[1] C. Baudrit and D. Dubois. Comparing methods for joint objective and subjective uncertainty propagation with an example in a risk assessment. In Proc. Fourth International Symposium on Imprecise Probabilities and Their Application (ISIPTA'05), pages 31-40, Pittsburg (USA, Pennsylvanie), 2005.

[2] S. Benferhat, D. Dubois, S. Kaci, and H. Prade. Bipolar possibility theory in preference modelling: representation, fusion and optimal solutions. Information Fusion, 7:135-150, 2006.

[3] G. Bordogna and G. Pasi. A fuzzy object oriented data model managing vague and uncertain information. International Journal of Intelligent Systems, 14(6):SCI 3495, 1999.

[4] P. Bosc, C. Brando, A. HadjAli, H. Jaudoin, and O. Pivert. Semantic proximity between queries and the empty answer problem. In IFSA/EUSFLAT Conf., pages 259-264, 2009.

[5] P. Bosc, A. HadjAli, and O. Pivert. Incremental controlled relaxation of failing flexible queries. J. Intell. Inf. Syst., 33(3):261-283, 2009.

[6] P. Bosc, L. Lietard, and O. Pivert. Soft querying, a new feature for database management system. In Proceedings DEXA'94 (Database and EXpert system Application), Lecture Notes in Computer Science \#856, pages 631-640. Springer-Verlag, 1994.

[7] P. Bosc, L. Lietard, and O. Pivert. Fuzziness in Database Management Systems, chapter Fuzzy theory techniques and applications in database management systems, pages 666-671. Academic Press, 1999. 
[8] P. Bosc and O. Pivert. SQLf: a relational database language for fuzzy querying. IEEE Transactions on fuzzy systems, 3(1):1-17, February 1995.

[9] P. Bosc and O. Pivert. About bipolar division operators. In Flexible Query Answering Systems, 8th International Conference, FQAS 2009, Roskilde, Denmark, October 26-28, 2009. Proceedings, volume 5822 of Lecture Notes in Computer Science, pages 572-582. Springer, 2009.

[10] P. Bosc, O. Pivert, A. Mokhtari, and L. Lietard. Extending relational algebra to handle bipolarity. In Proceedings of the 2010 ACM Symposium on Applied Computing (SAC), Sierre, Switzerland, March 22-26, 2010, pages 1718-1722. ACM, 2010.

[11] P. Bosc, O. Pivert, and G. Smits. A model based on outranking for database preference queries. In IPMU, pages 95-104, 2010.

[12] N. Bruno, S. Chaudhuri, and L. Gravano. Top-k selection queries over relational databases: Mapping strategies and performance evaluation. ACM Trans. Database Syst., 27(2):153-187, 2002.

[13] P. Buche and O. Haemmerlé. Towards a unified querying system of both structured and semi-structured imprecise data using fuzzy views. In Proceedings of the 8th International Conference on Conceptual Structures, Lecture Notes in Artificial Intelligence \#1867, pages 207220, Darmstadt, Germany, August 2000. Springer-Verlag.

[14] J. T. Cacioppo, W. L. Gardner, and G. G. Berntson. Beyond bipolar conceptualizations and measures: The case of attitudes \& evaluative space. Personality \& Social Psychology Review, 1:3-25, 1997.

[15] F. Charles, J. Sanchez, and N. Gontard. Modeling of active modified atmosphere packaging of endives exposed to several postharvest temperatures. Journal of Food Science, 8:443-448, 2005.

[16] J. Chomicki. Preference formulas in relational queries. ACM Trans. Database Syst., 28(4):427-466, 2003.

[17] S. Destercke, D. Dubois, and E. Chojnacki. Unifying practical uncertainty representations: II clouds. Int. J. of Approximate Reasoning (in press), 2007.

[18] S. Destercke, D. Dubois, and E. Chojnacki. Unifying practical uncertainty representations: I generalized p-boxes. Int. J. of Approximate Reasoning (In press), 2008.

[19] D. Dubois and H. Prade. Possibility Theory: An Approach to Computerized Processing of Uncertainty. Plenum Press, New York, 1988.

[20] D. Dubois and H. Prade. Fuzziness in Database Management Systems, chapter Tolerant fuzzy pattern matching: An introduction. PhysicaVerlag, 1995.

[21] D. Dubois and H. Prade. Bipolarity in flexible querying. In Flexible Query Answering Systems, 5th International Conference, FQAS 2002, 
Copenhagen, Denmark, October 27-29, 2002, Proceedings, volume 2522 of Lecture Notes in Computer Science, pages 174-182. Springer, 2002.

[22] D. Dubois and H. Prade. An overview of the asymetric bipolar representation of positive and negative information in possibility theory. Fuzzy Sets and System, 160:1355-1366, 2008.

[23] D. Dubois and H. Prade. An overview of the asymmetric bipolar representation of positive and negative information in possibility theory. Fuzzy Sets Syst., 160:1355-1366, 2009.

[24] W. Kießling and G. Köstler. Preference sql - design, implementation, experiences. In VLDB Proceedings of 28th International Conference on Very Large Data Bases, August 20-23, 2002, Hong Kong, China, pages 990-1001. Morgan Kaufmann, 2002.

[25] E. Klement, R. Mesiar, and E. Pap. Triangular Norms. Kluwer Academic Publisher, Dordrecht, 2000.

[26] P. Mahajan, F. Oliveira, J. Montanez, and J. Frias. Development of user-friendly software for design of modified atmosphere packaging for fresh and fresh-cut produce. Innovative Food Science and Emerging Technologies, 8:84-92, 2007.

[27] H. Prade. Lipski's approach to incomplete information data bases restated and generalized in the setting of Zadeh's possibility theory. Information Systems, 9(1):27-42, 1984.

[28] H. Prade and C. Testemale. Generalizing database relational algebra for the treatment of incomplete or uncertain information and vague queries. Information Sciences, 34:115-143, 1984.

[29] G. D. Tré, , and R. D. Caluwe. Recent research issues on the management of fuzziness in databases, chapter A generalized object-oriented database model, pages 155-182. Bordogna, G. and Pasi, G. (eds), Studies in Fuzziness and Soft computing, Vol. 53, Physica-Verlag, Heidelberg, Germany, 2000.

[30] G. D. Tré, S. Zadrozny, and A. Bronselaer. Handling bipolarity in elementary queries to possibilistic databases. IEEE Trans. on Fuzzy Systems, 18:599-612, 2010.

[31] G. D. Tré, S. Zadrozny, T. Matthé, J. Kacprzyk, and A. Bronselaer. Dealing with positive and negative query criteria in fuzzy database querying. In Flexible Query Answering Systems, 8th International Conference, FQAS 2009, Roskilde, Denmark, October 26-28, 2009. Proceedings, volume 5822 of Lecture Notes in Computer Science, pages 593-604. Springer, 2009.

[32] R. Yager. On ordered wieghted averaging aggregation operators in multicriteria decision making. IEEE Trans. on Syst., Man, and Cybern., 18:183-190, 1988. 
1079

1080

1081

1082

1083

1084

1085

1086

1087

1088

1089

1090

1091

1092

1093

1094

1095

1096

1097

1098

1099

1100

1101

1102

1103

1104

1105

1106

1107

1108

1109

1110

1111

1112

1113

1114

1115

1116

1117

1118

1119

1120

1121

1122

1123

1124

1125

1126

1127

[33] L. Zadeh. The concept of a linguistic variable and its application to approximate reasoning-i. Information Sciences, 8:199-249, 1975.

[34] L. Zadeh. Fuzzy sets as a basis for a theory of possibility. Fuzzy Sets and Systems, 1:3-28, 1978.

\section{UMR IATE}

E-mail address: sdestercke@gmail.com

UMR IATE

E-mail address: patrice.buche@supagro.inra.fr

UMR IATE

E-mail address: guillard@univ-montp2.fr 